\title{
Altered Temporal Dynamic Intrinsic Brain Activity in Late Blindness
}

\author{
Xin Huang, ${ }^{1}$ Zhi Wen, ${ }^{2}$ Chen-Xing Qi, ${ }^{1}$ Yan Tong $\mathbb{D}^{1}{ }^{1}$ Han-Dong Dan, ${ }^{1}$ Bao-Jun Xie $\mathbb{D},{ }^{2}$ \\ and Yin Shen iD 1,3 \\ ${ }^{1}$ Eye Center, Renmin Hospital of Wuhan University, Wuhan, 430060 Hubei, China \\ ${ }^{2}$ Department of Radiology, Renmin Hospital of Wuhan University, Wuhan, 430060 Hubei, China \\ ${ }^{3}$ Medical Research Institute, Wuhan University, Wuhan, Hubei, China
}

Correspondence should be addressed to Bao-Jun Xie; xiebj@126.com and Yin Shen; yinshen@whu.edu.cn

Received 1 January 2020; Revised 2 April 2020; Accepted 25 April 2020; Published 22 June 2020

Academic Editor: Maurizio Battaglia Parodi

Copyright (C) 2020 Xin Huang et al. This is an open access article distributed under the Creative Commons Attribution License, which permits unrestricted use, distribution, and reproduction in any medium, provided the original work is properly cited.

\begin{abstract}
Previous neuroimaging studies demonstrated that visual deprivation triggers significant crossmodal plasticity in the functional and structural architecture of the brain. However, prior neuroimaging studies focused on the static brain activity in blindness. It remains unknown whether alterations of dynamic intrinsic brain activity occur in late blindness (LB). This study investigated dynamic intrinsic brain activity changes in individuals with late blindness by assessing the dynamic amplitude of low-frequency fluctuations (dALFFs) using sliding-window analyses. Forty-one cases of late blindness (LB) (29 males and 12 females, mean age: $39.70 \pm 12.66$ years) and 48 sighted controls (SCs) (17 males and 31 females, mean age: $43.23 \pm 13.40$ years) closely matched in age, sex, and education level were enrolled in this study. The dALFF with sliding-window analyses was used to compare the difference in dynamic intrinsic brain activity between the two groups. Compared with SCs, individuals with LB exhibited significantly lower dALFF values in the bilateral lingual gyrus (LING)/calcarine (CAL) and left thalamus (THA). LB cases also showed considerably decreased dFC values between the bilateral LING/CAL and the left middle frontal gyrus (MFG) and between the left THA and the right LING/cerebelum_6 (CER) (two-tailed, voxel-level $P<0.01$, Gaussian random field (GRF) correction, cluster-level $P<0.05$ ). Our study demonstrated that LB individuals showed lower-temporal variability of dALFF in the visual cortices and thalamus, suggesting lower flexibility of visual thalamocortical activity, which might reflect impaired visual processing in LB individuals. These findings indicate that abnormal dynamic intrinsic brain activity might be involved in the neurophysiological mechanisms of LB.
\end{abstract}

\section{Introduction}

Visual deprivation leads to significant crossmodal plasticity in the brain's functional and structural architecture. Previous studies have demonstrated that visual deprivation triggers the visual cortex to other sensory processing, such as tactile [1], auditory [2], and olfactory functions [3], as well as higher cognitive functions (e.g., verbal memory [4] and episodic retrieval [5]). Furthermore, several neuroimaging studies have shown that blindness is associated with progressive atrophy of the visual pathway $[6,7]$ and visual cortices [8], as well as with abnormalities in non-visual areas $[9,10]$.

Recently, resting-state functional magnetic resonance imaging methods have been applied extensively to assess the effects of visual deprivation on the brain's functional architecture. Notably, visual deprivation has led to the reorganization of brain function. Liu et al. demonstrated that early blindness increases regional homogeneity ( $\mathrm{ReHo}$ ) in the visual cortex [11]. On their part, Jiang et al. reported that blindness increases regional spontaneous brain activity in visual areas and reduces it in sensorimotor and salience networks [12]. Visual deprivation has also been shown to induce significant abnormal interactions between the visual cortex and other sensory cortices. According to previous neuroimaging studies, blindness harbors abnormal interconnections between the visual cortex and other cortices: motor cortex $[13,14]$, Broca's area [15], and auditory cortex [16]. StriemAmit et al. found that central V1 is more strongly connected 
TABLE 1: Details of scanned parameters.

\begin{tabular}{lccc}
\hline Three-dimensional brain volume imaging (3D-BRAVO) & \multicolumn{2}{c}{ Gradient-echo-planar imaging sequence } \\
Repetition time/echo time & $8.5 / 3.3$ & Repetition time/echo time & $3.000 \mathrm{~ms} / 25 \mathrm{~ms}$ \\
\hline Slice thickness & $1.0 \mathrm{~mm}$ & Slice thickness & $1.2 \mathrm{~mm}$ \\
Acquisition matrix & $256 \times 256$ & Gap & $64 \times 64$ \\
Field of view & $240 \times 240 \mathrm{~mm} 2$ & Acquisition matrix & $90^{\circ}$ \\
Flip angle & $12^{\circ}$ & Flip angle & $240 \times 240 \mathrm{~mm} 2$ \\
& & Field of view & $3.6 \times 3.6 \times 3.6 \mathrm{~mm} 3$ \\
\hline
\end{tabular}

to language areas, whereas peripheral V1 is more powerfully associated with spatial attention and control networks in blindness [17]. Per Wang et al., congenital blindness has increased network connectivity within the salience network and occipital cortex, as well as abnormal internetwork connectivity between the salience network and the frontoparietal networks and sensorimotor networks [18]. However, these resting-state fMRI studies focused on static intrinsic brain activity and connectivity and did not assess the temporal dynamic intrinsic brain activity in late blindness. Recently, neuroimaging studies have begun focussing on investigating dynamic brain activity or networks that can reflect information on the variability in the strength or spatial dynamic organization of the brain $[19,20]$. Thus, we regard temporal dynamic brain activity analyses as a way to potentially deepen our understanding of brain activity changes in patients with blindness.

The human brain is a complex dynamic system capable of nonstationary neural activity and rapidly changing neural interaction. The human brain activity is inherently dynamic [21]. A map of the brain's dynamism reflects its temporal variability, which relates to the functional ability of neural networks [22]. Low-frequency oscillations $(<0.08 \mathrm{~Hz})$ of blood-oxygenation-level-dependent (BOLD) signaling in the human brain are physiologically meaningful. Notably, there is growing evidence that the temporal variability of BOLD signaling exists during the typical duration (a few minutes) of a resting-state scan of the human brain $[23,24]$. The temporal variability of BOLD signaling plays a critical role in the implementation of various physiological functions, such as consciousness [25] and cognition [26]. Sliding-window analysis and clustering methods have been used to investigate temporal variability in BOLD signaling $[27,28]$.

To study changes in BOLD signaling over time, slidingwindow correlation analysis, where the correlation is estimated for brain activity during multiple, possibly overlapping temporal segments (typically 30-60 s), has been widely deployed $[29,30]$. The amplitude of low-frequency fluctuations (ALFF) method is a reliable and sensitive functional magnetic resonance imaging technology for the quantification of local intrinsic brain activity [31]. Recently, the dynamic ALFF (dALFF) with a sliding-window analysis was successfully used to investigate the temporal variability of brain activity in patients with generalized tonic-clonic seizures [32], poststroke aphasia [33], and schizophrenia [34]. However, it is largely unknown whether dynamic spontane- ous brain activity changes occur in patients with blindness. Patients with retinitis pigmentosa (RP) offer a unique opportunity to study this issue. RP is an inherited retinal disease that primarily affects rod photoreceptor cells, followed by the degeneration of cone photoreceptor cells, eventually leading to blindness. Here, we selected RP patients who had experienced vision loss in adulthood. The goal of this study was to determine whether an altered dynamic spontaneous neural activity is present in blind patients. We hypothesized that blindness might be associated with abnormal dynamic spontaneous neural activity in vision and vision-related brain regions. Our findings may shed new light on the underlying pathological and compensatory mechanisms in blind patients.

\section{Materials and Methods}

2.1. Participants. Forty-one cases of late blindness (LB) (29 males and 12 females, mean age: $39.70 \pm 12.66$ years) and 48 sighted controls (SCs) (17 males and 31 females, mean age: $43.23 \pm 13.40$ years) participated in this study. All participants met the following criteria: (1) could be scanned with an MRI (e.g., no cardiac pacemaker or implanted metal devices); (2) did not have heart disease and claustrophobia; (3) did not have cerebral diseases (T1 images were checked by an experienced radiologist).

All LB subjects met the following criteria: (1) onset age of blindness > 12 years; (2) had no ocular surgical history.

All SC subjects met the following criteria: (1) had no ophthalmic diseases (glaucoma, optic neuritis, retinal degeneration, etc.); (2) had visual acuity $\geq 1.0$; (3) had no mental disorders.

Ethical statement: the study was approved by the medical research ethics committee and the institutional review board of the Renmin Hospital of Wuhan University Hospital. The protocol of the research followed the Declaration of Helsinki. All subjects provided written informed consent.

2.2. MRI Parameters. MRI scanning was performed on a $3-\mathrm{T}$ magnetic resonance scanner (Discovery MR 750W system; GE Healthcare, Milwaukee, WI, USA) with eight-channel head coil. All subjects underwent MRI scanning (eight minutes) with eyes closed without falling asleep and 240 functional images were obtained. The more details on scanning parameters were showed in Table 1. 
TABLE 2: Demographic measurements between the two groups.

\begin{tabular}{|c|c|c|c|c|}
\hline & LB group & SC group & $T$ values & $P$ values \\
\hline Gender (male/female) & $29 / 12$ & $17 / 31$ & 11.044 & 0.001 \\
\hline Age (years) & $39.70 \pm 12.66$ & $43.23 \pm 13.40$ & -1.267 & 0.208 \\
\hline Handedness & $41 \mathrm{R}$ & $48 \mathrm{R}$ & N/A & N/A \\
\hline Age of onset blindness (years) & $22.56 \pm 7.13$ & $\mathrm{~N} / \mathrm{A}$ & $\mathrm{N} / \mathrm{A}$ & $\mathrm{N} / \mathrm{A}$ \\
\hline
\end{tabular}

$\chi 2$ test for sex $(n)$. Independent $t$ test for the other normally distributed continuous data (means \pm SD). LB: late blindness; SC: sighted controls; N/A: not applicable.

2.3. fMRI Data Processing. The fMRI data preprocessing was performed using Data Processing \& Analysis of Brain Imaging toolbox (DPABI, http://www.rfmri.org/dpabi) [35], which is based on Statistical Parametric Mapping (SPM8) (http://www.fil.ion.ucl.ac.uk) implemented in MATLAB 2013a (MathWorks, Natick, MA, USA) and briefly the following steps [36]: (1) eliminate first ten time points for signal reaching equilibrium, and then slice timing and motion correction. For head motion parameters, more than $2 \mathrm{~mm}$ or for whom rotation exceeded $1.5^{\circ}$ during scanning were excluded. (2) Individual 3D-BRAVO images were registered to the mean fMRI data [37]. (3) Covariates (six head motion parameters, mean framewise displacement (FD), global brain signal, and the average signal from white matter signal and cerebrospinal fluid) were used to regress out. (4) Linear trends were removed and filtered $(0.01-0.08 \mathrm{~Hz})$. Scrubbing regression was not performed because contiguous time points were necessary for dynamic analysis [38].

2.4. dALFF Variance Computing. A sliding-window approach was used to compute the dALFF using the Dynamic Brain Connectome (DynamicBC) toolbox (v2.0, http://www.restfmri.net/forum/DynamicBC) [39]. For the sliding-window approach, to avoid the introduction of spurious fluctuations, the minimum window length should be larger than $1 / f \min$, where $f \min$ is the minimum frequency of the time series [40]. Here, a window length of 50 TR was considered as the optimal parameter to maintain the balance between capturing a rapidly shifting dynamic relationship and obtaining reliable estimates of the correlations between regions [41]. A window size of 50 TRs (100 s) and a window shifted by 10 TRs were selected [42]. Consequently, wholelength time courses were separated into 19 windows for each subject. An ALFF map was obtained for each sliding-window, and the ALFF of each voxel was standardized using $z$ -transformation.

2.5. dFC Variance Computing. The altered dALFF brain regions were identified as regions of interest (ROIs). 6- $\mathrm{mm}$ radii around the B-LING/CAL $[0,-72,6]$ and L-THA $[-6$, $-9,6$ ] coordinates were mapped as ROIs. A sliding-window approach via the DynamicBC toolbox (http://www.restfmri .net/forum/DynamicBC) was also used to obtain the wholebrain $\mathrm{dFC}$ maps of each seeded region. The variance of the time series of the correlation coefficient was estimated by calculating the standard deviation of $z$ values at each voxel to assess $\mathrm{dFC}$ flexibility.
2.6. Clinical Evaluation. Clinical data, including age, sex, and disease duration were recorded.

2.7. Statistical Analysis. The chi-square $\left(\chi^{2}\right)$ test and independent-sample $t$ test were performed to assess the behavioral data between two groups using SPSS version 20.0 (SPSS Inc, Chicago, IL, USA) $(P<0.05$ significant differences).

A one-sample $t$ test was conducted to assess intragroup patterns of zdALFF maps using the DPABI software. A two-sample $t$ test was used to assess zdALFF and the zdFC map difference between two groups' regressed covariates of age and sex and FD using the DPABI software. The Gaussian random field (GRF) method was used to correct for multiple comparisons (two-tailed, voxel-level $P<0.01$, GRF correction, cluster-level $P<0.05$ ).

Pearson correlation coefficient was used to assess the relationships between the dALFF and dFC values of different brain regions and clinical variables in the $\mathrm{LB}$ group using the SPSS version 20.0 software (SPSS Inc., Chicago, IL, USA).

2.8. Verification Analyses. To validate our dALFF findings, two different window lengths (30 TRs (60 s) and 100 TRs $(200 \mathrm{~s}))$ were calculated in the validation analysis. An ALFF map was obtained for each sliding window, and the dALFF of each voxel was standardized using $\mathrm{z}$-transformation.

\section{Results}

3.1. Demographic Measurements. There are no significant differences in age between two groups There are significant differences in gender $(P<0.001)$ between two groups. The age of onset blindness is $22.56 \pm 7.13$ years in the LB group. Details are shown in Table 2.

3.2. Dynamic ALFF Variance Differences. The spatial distribution of dALFF maps between the two groups is shown in Figure 1. Compared with SCs, individuals with LB exhibited significantly lower dALFF values in the bilateral LING/CAL and left THA (Figure 2(a) (blue) and Table 3). The mean values of altered dALFF between the two groups are shown in Figure 2(b).

3.3. Dynamic FC Variance Differences. Compared with SCs, LB cases exhibited markedly decreased dFC values between the bilateral LING/CAL and the left MFG and between the left THA and the right LING/CER (Figures 3(a) and 3(b) (blue) and Table 4). The mean values of altered $\mathrm{dFC}$ readings between the two groups are shown in Figures 3(c) and 3(d). 


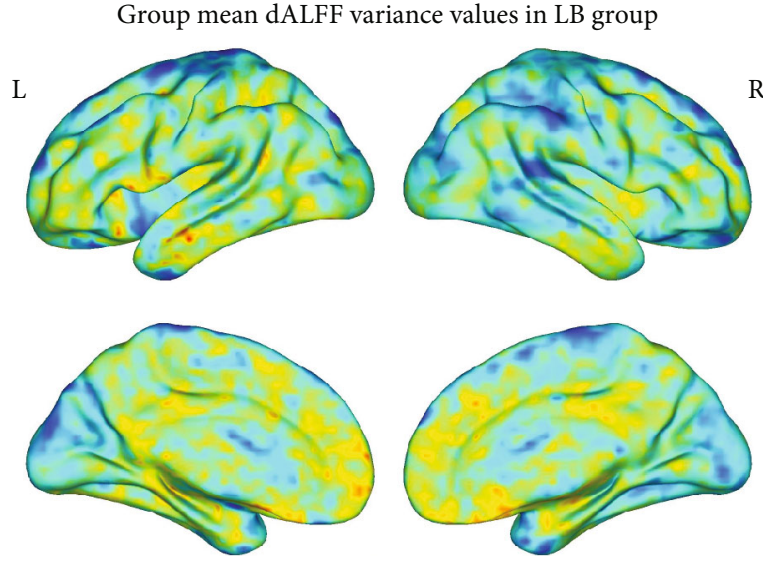

(a)
Group mean dALFF variance values in SC group
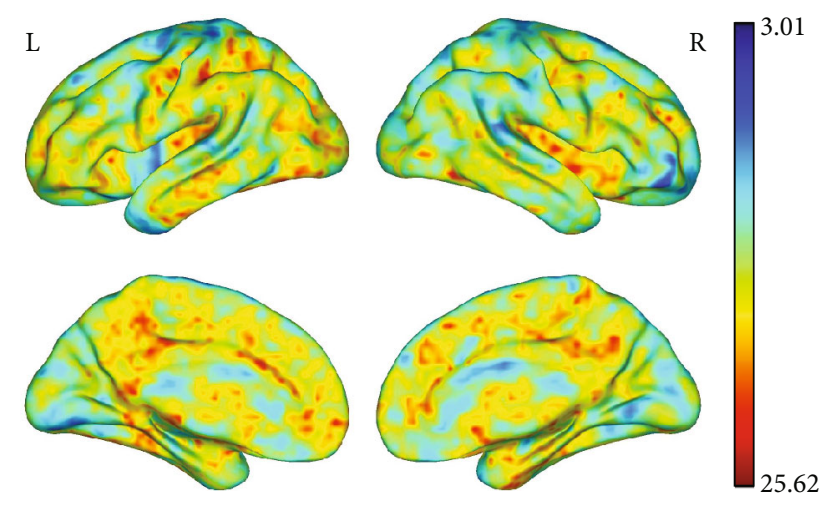

(b)

FIgURE 1: Spatial patterns of dALFF variance were observed at the group level in LB and SC groups in the typical frequency band (0.01$0.08 \mathrm{~Hz}$ ). Within group mean dALFF variance maps within the LB (a) and SC (b). dALFF: dynamic amplitude of low-frequency fluctuation; LB: late blindness; SC: sighted controls; L: left; R: right.

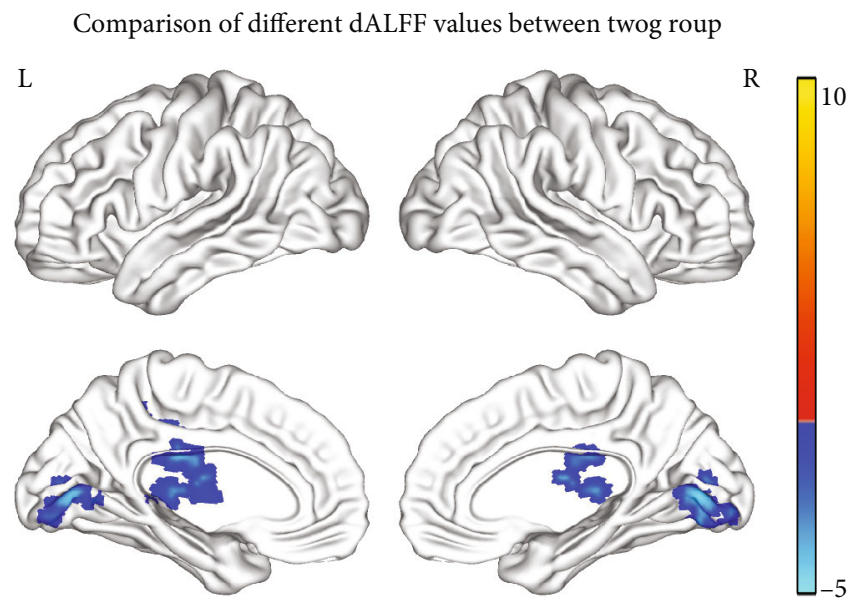

(a)

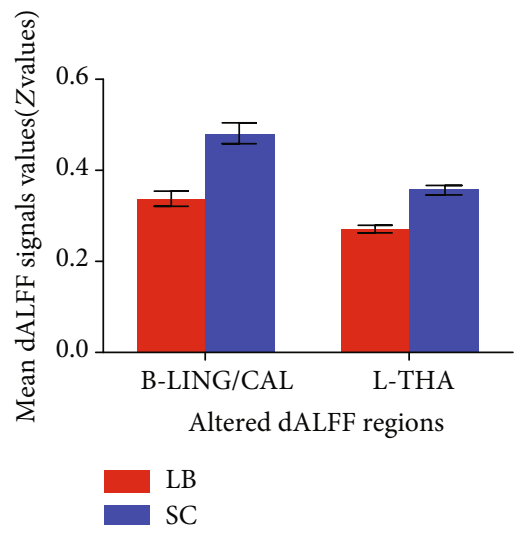

(b)

FIGURE 2: Comparison of different dALFF values between LB group and SC group. Significant dALFF values differences were observed in the B-LING/CAL, L-THA. The blue areas indicate lower dALFF values (voxel-level $P<0.01$, GRF correction, cluster-level $P<0.05)(\mathbf{a})$. The mean values of altered dALFF values between the LB and SC groups (b). dALFF: dynamic amplitude of low-frequency fluctuation; LB: late blindness; SC: sighted controls; GRF: Gaussian random field; LING: lingual gyrus; CAL: calcarine; THA: thalamus; L: left; B: bilateral;.

3.4. Receiver Operating Characteristic Curve. To test the sensitivity and specificity of dALFF and $\mathrm{dFC}$ value differences between the two groups, the areas under the ROC curve for dALFF were $\mathrm{LB}<\mathrm{HC}$, for bilateral LING/CAL, 0.770 $(P<0.001 ; 95 \%$ CI: 0.671-0.920); for left THA, 0.843 $(P<0.001 ; 95 \% \mathrm{CI}: 0.762-0.925)$; (Figure 4(a)). The areas under the ROC curve for $\mathrm{dFC}$ were $\mathrm{LB}<\mathrm{HC}$, for left $\mathrm{MFG}$, 0.836 ( $P<0.001$; 95\% CI: 0.752-0.920); for right LING/CER, 0.806 ( $P<0.001$; 95\% CI: 0.714-0.898); (Figure 4(b)).

3.5. Verification Analyses. In the verification analyses, we found that the group differences in dALFF variability with different window lengths (30 TRs (60s) and 100 TRs (200s)) were similar to those of the main findings. Detailed information is presented in the Supplementary Materials. In the 30 TRs window length step analyses, the LB group had significantly decreased dALFF values in the bilateral
LING/CAL and left THA, compared with the SC group (Figure S1 and Table S1). Meanwhile, in the 100 TRs window length step analyses, the LB group had substantially decreased dALFF values in the bilateral CAL, compared with the SC group (Figure S2 and Table S1).

\section{Discussion}

Our study is the first of its kind to investigate dynamic spontaneous neural activity changes in LB using dALFF with sliding-window analyses. We showed that individuals with LB displayed significantly lower dALFF values in the bilateral LING/CAL and left THA relative to the SC group. Also, the LB group showed remarkably lower $\mathrm{dFC}$ values between the bilateral LING/CAL and the left MFG, as well as between the left THA and the right LING/CER relative to the SC group. 
TABLE 3: Significant differences in the dALFF between the two groups.

\begin{tabular}{|c|c|c|c|c|c|c|c|}
\hline \multirow{2}{*}{\multicolumn{2}{|c|}{ Condition/brain regions }} & \multirow{2}{*}{$\mathrm{BA}$} & \multirow{2}{*}{ Peak $T$ scores } & \multicolumn{3}{|c|}{ MNI coordinates } & \multirow{2}{*}{ Cluster size (voxels) } \\
\hline & & & & $x$ & $y$ & $z$ & \\
\hline \multicolumn{8}{|c|}{ ROI in B-LING/CAL } \\
\hline $\mathrm{LB}<\mathrm{SC}$ & L-MFG & 8 & -4.2625 & -39 & 12 & 48 & 83 \\
\hline \multicolumn{8}{|c|}{ ROI in L-THA } \\
\hline $\mathrm{LB}<\mathrm{SC}$ & R-LING/CER & - & -4.1493 & 9 & -66 & -15 & 45 \\
\hline
\end{tabular}

The statistical threshold was set at the voxel level with $P<0.01$ for multiple comparisons using the Gaussian random field theory (voxel-level $P<0.01$, GRF correction, cluster-level $P<0.05)$. dALFF: dynamic amplitude of low-frequency fluctuation; LB: late blindness; SC: sighted control; LING: lingual gyrus; CAL: calcarine; THA: thalamus; B: bilateral; L: left; GRF: Gaussian random field.

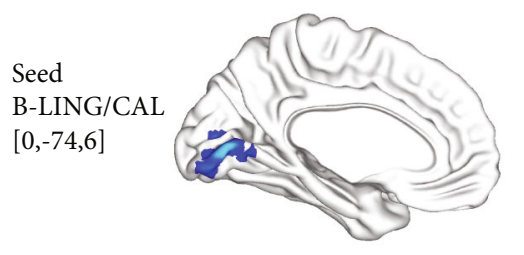

$\mathrm{L}$
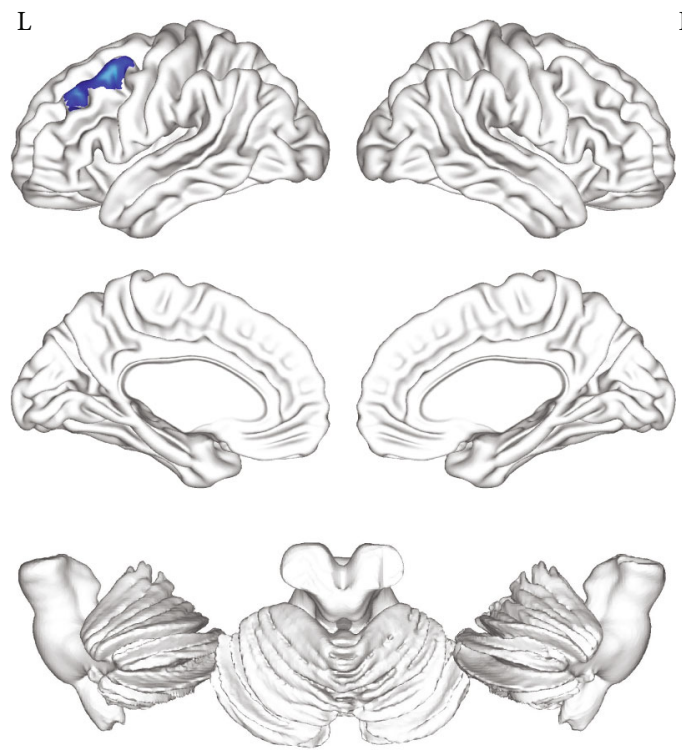

(a)

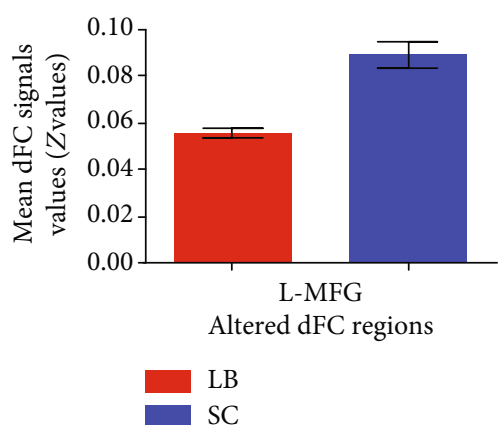

(c)

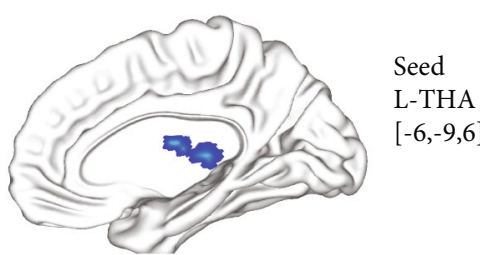

R L
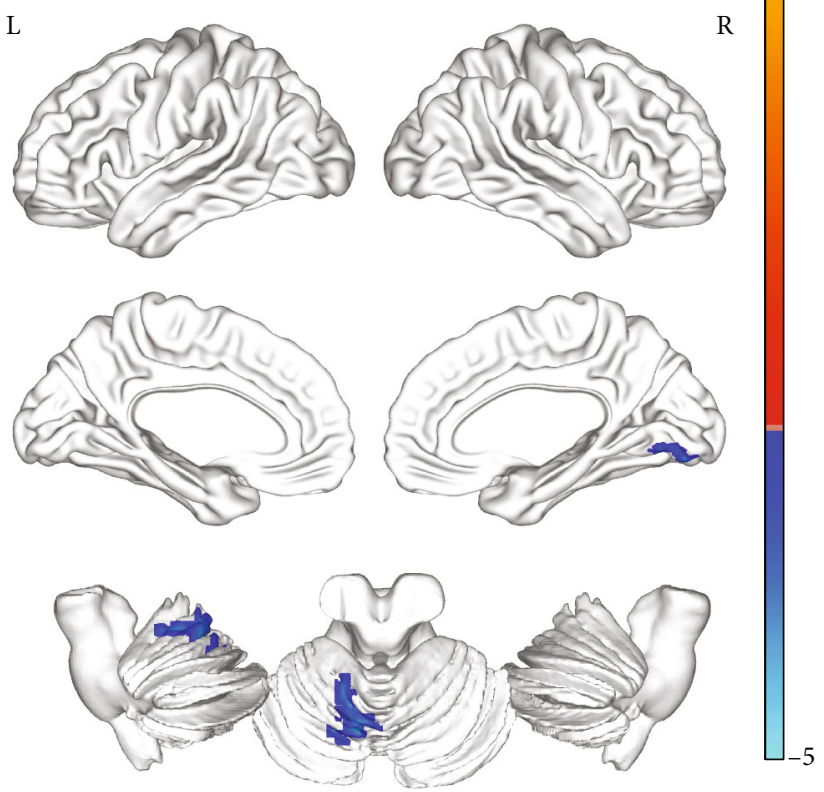

(b)

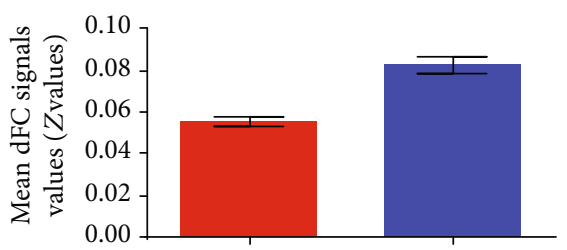

R-LING/CER

Altered dFC regions

(d)

FIGURE 3: Comparison of different dFC values between LB group and SC group. Significant dFC values differences were observed in the LMFG (a) and R-LING/CER (b). The blue areas indicate lower dFC values.(voxel-level $P<0.01$, GRF correction, cluster-level $P<0.05$ ) The mean values of altered $\mathrm{dFC}$ values between the LB and SC groups. (c, d). dFC: dynamic functional connectivity; LB: late blindness; SC: sighted controls; GRF: Gaussian random field; MFG: middle frontal gyrus; LING: lingual gyrus; CER: cerebelum_6; L: left; R: right. 
TABLE 4: Significant differences in dFC values between the two groups.

\begin{tabular}{|c|c|c|c|c|c|c|c|}
\hline \multirow{2}{*}{\multicolumn{2}{|c|}{ Condition/brain regions }} & \multirow{2}{*}{ BA } & \multirow{2}{*}{ Peak $T$ scores } & \multicolumn{3}{|c|}{ MNI coordinates } & \multirow{2}{*}{ Cluster size (voxels) } \\
\hline & & & & $x$ & $y$ & $z$ & \\
\hline $\mathrm{LB}<\mathrm{SC}$ & B-LING/CAL & 18 & -4.5301 & 0 & -72 & 6 & 177 \\
\hline $\mathrm{LB}<\mathrm{SC}$ & L-THA & 18 & -3.7118 & -6 & -9 & 6 & 105 \\
\hline
\end{tabular}

The statistical threshold was set at the voxel level with $P<0.01$ for multiple comparisons using the Gaussian random field theory (voxel-level $P<0.01$, GRF correction, cluster-level $P<0.05)$. dFC: dynamic functional connectivity; BA: Brodmann area; LB: late blindness; SC: sighted control; MNI: Montreal Neurological Institute; GRF: Gaussian random field; LING: lingual gyrus; CAL: calcarine; THA: thalamus; MFG: middle frontal gyrus; CER: cerebelum_6.

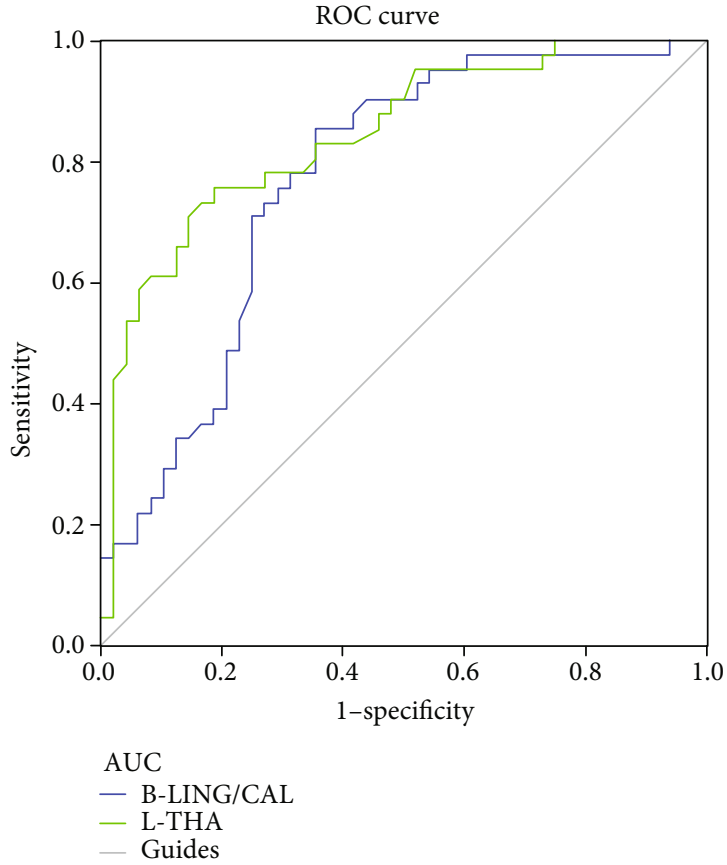

(a)

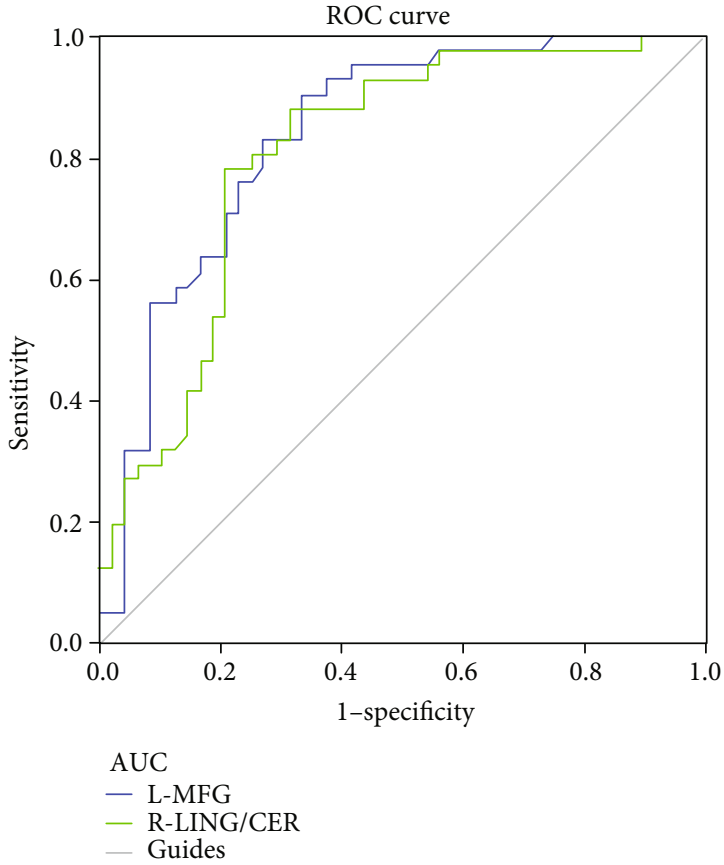

(b)

Figure 4: ROC curve analysis of the mean dALFF and dFC of altered brain regions. ROC curve in dALFF values: $\mathrm{LB}<\mathrm{HC}$, for B-LING/CAL, $0.770(P<0.001$; 95\% CI: 0.671-0.920); for L-THA, $0.843(P<0.001$; 95\% CI: 0.762-0.925) (a); ROC curve in dFC values: LB<HC, for LMFG, $0.836(P<0.001$; 95\% CI: 0.752-0.920); for R-LING/CER, $0.806(P<0.001$; 95\% CI: 0.714-0.898) (b). ROC: receiver operating characteristic; dALFF: amplitude of low-frequency fluctuation; AUC: area under the curve; LING: lingual gyrus; CAL: calcarine; THA: thalamus; MFG: middle frontal gyrus; CER: cerebelum_6; L: left; R: right; B: bilateral;.

The bilateral LING/CAL is the location of the primary visual cortex in the human brain, which receives visual signals from the visual pathway and transfers them to higher visual cortices. In our previous study, we demonstrated that RP patients had significantly lower ALFF values in the bilateral lingual gyrus/cerebellum posterior lobe relative to the HC group [43]. RP patients also had considerably lower regional homogeneity values in the bilateral lingual gyrus/cerebellum posterior lobe [44]. Hou et al. found that blind patients showed reduced voxel-mirrored homotopic connectivity in the primary visual cortex and visual association cortex, compared with SCs [45]. Qin et al., meanwhile, demonstrated that patients with congenital blindness (CB) and late blindness (LB) had reduced short- and long-range functional connectivity density in the primary visual cortex relative to the SC group [46]. Consistent with these findings, our study revealed that individuals in the LB group had significantly lower dALFF values in the bilateral LING/CAL relative to the SC group. Flexibility in spontaneous neural activity has been associated with behaviorally advantageous changes in brain network dynamics [47]. Thus, our results suggest that reduced flexibility of the brain's activity in the LING/CAL might reflect impaired visual processing in people with LB.

Additionally, we found that LB persons displayed significantly lower dFC values between the bilateral LING/CAL and the left MFG; the frontal lobe was closely linked to higher cognitive function. The MFG is involved in executive attention [48], language [49], and emotion [50]. Previous neuroimaging studies reported robust correlations between the visual cortex and frontal lobe, involving vision-for-action [51] and visuomotor functions [52]. Our results here suggest that lower flexibility of FC between the bilateral LING/CAL and the left MFG might reflect impaired vision-for-action in people with LB.

Remarkably, this study established that individuals with LB had lower dALFF values in the left THA and lower dFC between the left THA and right LING/CER relative to the 
SC group. The THA is an important subcortical nucleus that transfers various afferents from multiple sensory organs to the primary sensory cortex [53]. Karlen et al. demonstrated that early blindness induces abnormal patterns in thalamocortical and corticocortical connections [54], and Ptito et al. observed abnormalities in the structures of the retinothalamocortical pathway in patients with congenital blindness [55]. Another study revealed that patients with blindness displayed significant alterations in the thalamic microstructure [56]. Consistent with these findings, we speculated that a reduced visual signal input due to blindness might cause the dysfunction of the thalamus. Our results revealed that reduced flexibility of the brain's activity in the left THA might reflect an impaired retinothalamocortical pathway in LB persons.

There are some limitations to this study. First, the selection of the sliding-window length remains a subject of debate. We selected $50 \mathrm{TR}$ as the window length based on the criterion that the minimum length should be more than $1 / f \mathrm{~min}$. The results of different sliding-window lengths were similar to those of the main results with 50 TR, suggesting that our findings on dALFF were relatively stable. Second, our study used relatively small sample sizes. We intend to use larger sample sizes in the future. Third, the LB group exhibited different ages of blindness onset, which might be associated with heterogeneity in the presentation of LB. Some covariates, including age and gender, meanwhile, were regressed in the statistical analysis to reduce their impact on the accuracy of results. In terms of statistical methods, FDR correction may be used to reduce the bias of results in future studies. Besides, dynamic functional network connectivity method would be used to further reveal the changes in neural mechanisms of blindness in the future study.

\section{Conclusion}

We have shown that individuals with LB exhibited lower temporal variability of dALFF in the visual cortices and thalamus, suggesting lower flexibility in visual thalamocortical activity, which might reflect impaired visual processing in these patients. These findings indicate that abnormal dynamic spontaneous brain activity might be involved in the pathophysiological mechanisms of LB.

\section{Data Availability}

The datasets used and/or analyzed during the current study are available from the corresponding author on reasonable request.

\section{Ethical Approval}

The study was approved by the Renmin Hospital of Wuhan University Hospital Institutional Review Committee on Human Research.

\section{Consent}

All of the participants and their authorized caregivers provided written informed consent.

\section{Conflicts of Interest}

The authors declare that they have no conflicts of interest.

\section{Authors' Contributions}

X.H., Z.W., and B.J.X. Y.S. contributed to data collection and statistical analyses and wrote the manuscript. Y.S. designed the protocol and reviewed and edited the manuscript. Y.S., X.H., Z.W., Y.T., C.X.Q., and H.D.D designed the protocol and contributed to MRI analysis. Y.T., C.X.Q., and H.D.D. designed the study and oversaw all clinical aspects of study conduct and manuscript preparation. Xin Huang and Zhi Wen contributed equally to this work.

\section{Acknowledgments}

This research was supported by The National Nature Science Foundation of China (Grant No. 81800872); International Science \& Technology Cooperation Program of China (2017YFE0103400).

\section{Supplementary Materials}

Figure S1: comparison of different dALFF values between LB group and SC group (a window size of 30 TRs (60 s), and window shifted by 10 TRs (20 s)). Figure S2: comparison of different dALFF values between LB group and SC group (a window size of 100 TRs ( $200 \mathrm{~s}$ ), and window shifted by 10 TRs (20 s)). Table S1: significant differences in the dALFF between two groups. (Supplementary materials)

\section{References}

[1] N. Sadato, A. Pascual-Leone, J. Grafman et al., "Activation of the primary visual cortex by Braille reading in blind subjects," Nature, vol. 380, no. 6574, pp. 526-528, 1996.

[2] K. E. Watkins, T. J. Shakespeare, M. C. O'Donoghue et al., "Early auditory processing in area V5/MT+ of the congenitally blind brain," The Journal of Neuroscience, vol. 33, no. 46, pp. 18242-18246, 2013.

[3] R. Kupers, M. Beaulieu-Lefebvre, F. C. Schneider et al., "Neural correlates of olfactory processing in congenital blindness," Neuropsychologia, vol. 49, no. 7, pp. 2037-2044, 2011.

[4] A. Amedi, N. Raz, P. Pianka, R. Malach, and E. Zohary, "Early 'visual' cortex activation correlates with superior verbal memory performance in the blind," Nature Neuroscience, vol. 6, no. 7, pp. 758-766, 2003.

[5] N. Raz, A. Amedi, and E. Zohary, "V1 activation in congenitally blind humans is associated with episodic retrieval," Cereb Cortex, vol. 15, no. 9, pp. 1459-1468, 2005.

[6] N. L. Reislev, R. Kupers, H. R. Siebner, M. Ptito, and T. B. Dyrby, "Blindness alters the microstructure of the ventral but not the dorsal visual stream," Brain Structure \& Function, vol. 221, no. 6, pp. 2891-2903, 2016. 
[7] W. J. Pan, G. Wu, C. X. Li, F. Lin, J. Sun, and H. Lei, "Progressive atrophy in the optic pathway and visual cortex of early blind Chinese adults: a voxel-based morphometry magnetic resonance imaging study," NeuroImage, vol. 37, no. 1, pp. 212-220, 2007.

[8] Q. Li, M. Song, J. Xu, W. Qin, C. Yu, and T. Jiang, “Cortical thickness development of human primary visual cortex related to the age of blindness onset," Brain Imaging and Behavior, vol. 11, no. 4, pp. 1029-1036, 2017.

[9] T. Elbert, A. Sterr, B. Rockstroh, C. Pantev, M. M. Müller, and E. Taub, "Expansion of the tonotopic area in the auditory cortex of the blind," The Journal of Neuroscience, vol. 22, no. 22, pp. 9941-9944, 2002.

[10] N. Leporé, P. Voss, F. Lepore et al., "Brain structure changes visualized in early- and late-onset blind subjects," NeuroImage, vol. 49, no. 1, pp. 134-140, 2010.

[11] C. Liu, Y. Liu, W. Li et al., "Increased regional homogeneity of blood oxygen level-dependent signals in occipital cortex of early blind individuals," Neuroreport, vol. 22, no. 4, pp. 190194, 2011.

[12] A. Jiang, J. Tian, R. Li et al., "Alterations of regional spontaneous brain activity and gray matter volume in the blind," Neural Plasticity, vol. 2015, Article ID 141950, 12 pages, 2015.

[13] Z. Wen, F. Q. Zhou, X. Huang, H. D. Dan, B. J. Xie, and Y. Shen, "Altered functional connectivity of primary visual cortex in late blindness," Neuropsychiatric Disease and Treatment, vol. Volume 14, pp. 3317-3327, 2018.

[14] C. Yu, Y. Liu, J. Li et al., "Altered functional connectivity of primary visual cortex in early blindness," Human Brain Mapping, vol. 29, no. 5, pp. 533-543, 2008.

[15] N. Sabbah, C. N. Authié, N. Sanda et al., "Increased functional connectivity between language and visually deprived areas in late and partial blindness," NeuroImage, vol. 136, pp. 162173, 2016.

[16] M. Pelland, P. Orban, C. Dansereau, F. Lepore, P. Bellec, and O. Collignon, "State-dependent modulation of functional connectivity in early blind individuals," NeuroImage, vol. 147, pp. 532-541, 2017.

[17] E. Striem-Amit, S. Ovadia-Caro, A. Caramazza, D. S. Margulies, A. Villringer, and A. Amedi, "Functional connectivity of visual cortex in the blind follows retinotopic organization principles," Brain, vol. 138, no. 6, pp. 1679-1695, 2015.

[18] D. Wang, W. Qin, Y. Liu, Y. Zhang, T. Jiang, and C. Yu, "Altered resting-state network connectivity in congenital blind," Human Brain Mapping, vol. 35, no. 6, pp. 2573-2581, 2014.

[19] J. Li, X. Duan, Q. Cui, H. Chen, and W. Liao, "More than just statics: temporal dynamics of intrinsic brain activity predicts the suicidal ideation in depressed patients," Psychological Medicine, vol. 49, no. 5, pp. 852-860, 2019.

[20] W. Liao, H. Chen, J. Li et al., "Endless Fluctuations: Temporal Dynamics of the Amplitude of Low Frequency Fluctuations," IEEE Transactions on Medical Imaging, vol. 38, no. 11, pp. 2523-2532, 2019.

[21] V. D. Calhoun, R. Miller, G. Pearlson, and T. Adal1, "The chronnectome: time-varying connectivity networks as the next frontier in fMRI data discovery," Neuron, vol. 84, no. 2, pp. 262-274, 2014.

[22] J. Kim, M. Criaud, S. S. Cho et al., "Abnormal intrinsic brain functional network dynamics in Parkinson's disease," Brain, vol. 140, no. 11, pp. 2955-2967, 2017.
[23] F. Mokhtari, M. I. Akhlaghi, S. L. Simpson, G. Wu, and P. J. Laurienti, "Sliding window correlation analysis: Modulating window shape for dynamic brain connectivity in resting state," NeuroImage, vol. 189, pp. 655-666, 2019.

[24] Z. Ma and N. Zhang, "Temporal transitions of spontaneous brain activity," Elife, vol. 7, 2018.

[25] F. Cavanna, M. G. Vilas, M. Palmucci, and E. Tagliazucchi, "Dynamic functional connectivity and brain metastability during altered states of consciousness," Neuroimage, vol. 180, pp. 383-395, 2018.

[26] P. Wang, R. Li, B. Liu et al., "Altered static and temporal dynamic amplitude of low-frequency fluctuations in the background network during working memory states in mild cognitive impairment," Frontiers in Aging Neuroscience, vol. 11, 2019.

[27] U. Sakoğlu, G. D. Pearlson, K. A. Kiehl, Y. M. Wang, A. M. Michael, and V. D. Calhoun, "A method for evaluating dynamic functional network connectivity and task-modulation: application to schizophrenia," Magma, vol. 23, no. 5-6, pp. 351-366, 2010.

[28] B. M. Baczkowski, T. Johnstone, H. Walter, S. Erk, and I. M. Veer, "Sliding-window analysis tracks fluctuations in amygdala functional connectivity associated with physiological arousal and vigilance during fear conditioning," NeuroImage, vol. 153, pp. 168-178, 2017.

[29] E. A. Allen, E. Damaraju, S. M. Plis, E. B. Erhardt, T. Eichele, and V. D. Calhoun, "Tracking whole-brain connectivity dynamics in the resting state," Cerebral Cortex, vol. 24, no. 3, pp. 663-676, 2014.

[30] C. Chang and G. H. Glover, "Time-frequency dynamics of resting-state brain connectivity measured with fMRI," Neuroimage, vol. 50, no. 1, pp. 81-98, 2010.

[31] Y. F. Zang, Y. He, C. Z. Zhu et al., "Altered baseline brain activity in children with ADHD revealed by resting- state functional MRI," Brain and Development, vol. 29, no. 2, pp. 8391, 2007.

[32] H. Liu, W. Li, M. Zhao et al., "Altered temporal dynamics of brain activity in patients with generalized tonic-clonic seizures," PLoS One, vol. 14, no. 7, 2019.

[33] J. Guo, B. B. Biswal, S. Han et al., "Altered dynamics of brain segregation and integration in poststroke aphasia," Human Brain Mapping, vol. 40, no. 11, pp. 3398-3409, 2019.

[34] S. Yang, Y. Meng, J. Li et al., “Temporal dynamic changes of intrinsic brain activity in schizophrenia with cigarette smoking," Schizophrenia Research, vol. 210, pp. 66-72, 2019.

[35] C. G. Yan, X. D. Wang, X. N. Zuo, and Y. F. Zang, "DPABI: data processing \& analysis for (resting-state) brain imaging," Neuroinformatics, vol. 14, no. 3, pp. 339-351, 2016.

[36] S. Yin, L. Liu, J. Tan, C. Ding, D. Yao, and A. Chen, “Attentional control underlies the perceptual load effect: evidence from voxel-wise degree centrality and resting-state functional connectivity," Neuroscience, vol. 362, pp. 257-264, 2017.

[37] Japanese Alzheimer's Disease Neuroimaging Initiative, M. Goto, O. Abe et al., "Diffeomorphic Anatomical Registration Through Exponentiated Lie Algebra provides reduced effect of scanner for cortex volumetry with atlas-based method in healthy subjects," Neuroradiology, vol. 55, no. 7, pp. 869875, 2013.

[38] C. G. Yan, B. Cheung, C. Kelly et al., "A comprehensive assessment of regional variation in the impact of head micromovements on functional connectomics," NeuroImage, vol. 76, pp. 183-201, 2013. 
[39] W. Liao, G. R. Wu, Q. Xu et al., "DynamicBC: a MATLAB toolbox for dynamic brain connectome analysis," Brain Connectivity, vol. 4, no. 10, pp. 780-790, 2014.

[40] N. Leonardi and D. Van De Ville, "On spurious and real fluctuations of dynamic functional connectivity during rest," NeuroImage, vol. 104, pp. 430-436, 2015.

[41] Q. Cui, W. Sheng, Y. Chen et al., "Dynamic changes of amplitude of low-frequency fluctuations in patients with generalized anxiety disorder," Human Brain Mapping, vol. 41, no. 6, pp. 1667-1676, 2020.

[42] W. Liao, J. Li, X. Duan, Q. Cui, H. Chen, and H. Chen, "Static and dynamic connectomics differentiate between depressed patients with and without suicidal ideation," Human Brain Mapping, vol. 39, no. 10, pp. 4105-4118, 2018.

[43] X. Huang, F. Q. Zhou, H. D. Dan, and Y. Shen, “Abnormal intrinsic brain activity in individuals with peripheral vision loss because of retinitis pigmentosa using amplitude of lowfrequency fluctuations," Neuroreport, vol. 29, no. 15, pp. 1323-1332, 2018.

[44] H. D. Dan, F. Q. Zhou, X. Huang, Y. Q. Xing, and Y. Shen, "Altered intra- and inter-regional functional connectivity of the visual cortex in individuals with peripheral vision loss due to retinitis pigmentosa," Vision Research, vol. 159, pp. $68-75,2019$.

[45] F. Hou, X. Liu, Z. Zhou, J. Zhou, and H. Li, "Reduction of interhemispheric functional brain connectivity in early blindness: a resting-state fMRI study," BioMed Research International, vol. 2017, Article ID 6756927, 8 pages, 2017.

[46] W. Qin, Y. Xuan, Y. Liu, T. Jiang, and C. Yu, "Functional connectivity density in congenitally and late blind subjects," Cerebral Cortex, vol. 25, no. 9, pp. 2507-2516, 2015.

[47] U. Braun, A. Schäfer, H. Walter et al., "Dynamic reconfiguration of frontal brain networks during executive cognition in humans," Proceedings of the National Academy of Sciences of the United States of America, vol. 112, no. 37, pp. 1167811683, 2015.

[48] M. Andersson, M. Ystad, A. Lundervold, and A. J. Lundervold, "Correlations between measures of executive attention and cortical thickness of left posterior middle frontal gyrus - a dichotic listening study," Behavioral and Brain Functions, vol. 5, no. 1, p. 41, 2009.

[49] J. Sierpowska, A. Fernandez-Coello, A. Gomez-Andres et al., "Involvement of the middle frontal gyrus in language switching as revealed by electrical stimulation mapping and functional magnetic resonance imaging in bilingual brain tumor patients," Cortex, vol. 99, pp. 78-92, 2018.

[50] J. Y. Park, B. M. Gu, D. H. Kang et al., "Integration of crossmodal emotional information in the human brain: an fMRI study," Cortex, vol. 46, no. 2, pp. 161-169, 2010.

[51] R. Caminiti, S. Ferraina, and P. B. Johnson, "The sources of visual information to the primate frontal lobe: a novel role for the superior parietal lobule," Cerebral Cortex, vol. 6, no. 3, pp. 319-328, 1996.

[52] D. Boussaoud, G. di Pellegrino, and S. P. Wise, "Frontal lobe mechanisms subserving vision-for-action versus vision-forperception," Behavioural Brain Research, vol. 72, no. 1-2, pp. 1-15, 1995.

[53] I. C. Lin, D. Xing, and R. Shapley, "Integrate-and-fire vs Poisson models of LGN input to V1 cortex: noisier inputs reduce orientation selectivity," Journal of Computational Neuroscience, vol. 33, no. 3, pp. 559-572, 2012.
[54] S. J. Karlen, D. M. Kahn, and L. Krubitzer, "Early blindness results in abnormal corticocortical and thalamocortical connections," Neuroscience, vol. 142, no. 3, pp. 843-858, 2006.

[55] M. Ptito, F. C. Schneider, O. B. Paulson, and R. Kupers, "Alterations of the visual pathways in congenital blindness," Experimental Brain Research, vol. 187, no. 1, pp. 41-49, 2008.

[56] N. H. Reislev, T. B. Dyrby, H. R. Siebner, H. Lundell, M. Ptito, and R. Kupers, "Thalamocortical connectivity and microstructural changes in congenital and late blindness," Neural Plasticity, vol. 2017, Article ID 9807512, 11 pages, 2017. 